\title{
Codimension one symplectic foliations
}

Omegar Calvo, Vicente Muñoz and Francisco Presas

\begin{abstract}
We define the concept of symplectic foliation on a symplectic manifold and provide a method of constructing many examples, by using asymptotically holomorphic techniques.
\end{abstract}

\section{Introduction}

During the last three decades there has been an active field of research related to the study of holomorphic foliations over a complex manifold $[5,7,10,13]$. To define a codimension one holomorphic foliation we need to fix a holomorphic line bundle $L$ over the complex manifold $M$. Then we choose a non-zero holomorphic section $\alpha \in H^{0}\left(M, T^{*} M \otimes L\right)$, satisfying the integrability condition:

$$
\alpha \wedge d \alpha=0
$$

There is an equivalence relation given by multiplication of $\alpha$ by no-where zero holomorphic functions, and a holomorphic foliation is defined as an equivalence class of such integrable 1-forms. In what follows we restrict ourselves to the case where $M$ is compact, so that the set of foliations is a subset in the projective space $\mathbb{P} H^{0}\left(M, T^{*} M \otimes L\right)$.

In this work, we aim to generalize this notion to the symplectic category. We give the following definition:

Definition 1.1 A symplectic foliation $\alpha$ with normal line bundle $L$ on a symplectic manifold $(M, \omega)$ is a non-zero element of $\mathcal{C}^{\infty}\left(T_{\mathbb{C}}^{*} M \otimes_{\mathbb{C}} L\right)$ which satisfies the integrability condition (1.1). Also we impose that the set of singularities, defined as $S_{\alpha}=\{x \in M \mid \alpha(x)=0\}$, is a finite union of

2000 Mathematics Subject Classification: Primary: 53D35. Secondary: 37F75, 53C12. Keywords: Foliation, symplectic, asymptotically holomorphic. 
symplectic submanifolds of real codimension greater than or equal to four and whose intersections are transverse and symplectic. Finally, we impose that for any $p \in M-S_{\alpha}$ the subspace $\operatorname{ker} \alpha(p) \subset T_{p} M$ is symplectic.

Two symplectic foliations $\alpha_{1}$ and $\alpha_{2}$ are considered equivalent whenever there is an isomorphism $\psi: L \rightarrow L$ as real plane bundles such that $\psi^{*} \alpha_{2}=\alpha_{1}$.

To understand $\operatorname{ker} \alpha(p)$ as a subspace of $T_{p} M$, we look at the isomorphism $T_{\mathbb{C}}^{*} M \otimes_{\mathbb{C}} L=T^{*} M \otimes_{\mathbb{R}} L$, where $T_{\mathbb{C}}^{*} M$ is the complexified cotangent bundle. Therefore we may interpret $\alpha(p): T_{p} M \rightarrow L_{p}$ as a real linear map and ker $\alpha(p) \subset T_{p} M$ is a codimension two subspace.

Now if $\alpha_{1}$ and $\alpha_{2}$ are equivalent then $S_{\alpha_{1}}=S_{\alpha_{2}}$ and the topological foliations coincide $\operatorname{ker} \alpha_{1}=\operatorname{ker} \alpha_{2}$. Note that the isomorphism $\psi: L \rightarrow L$ takes values in $\operatorname{GL}(2, \mathbb{R})$, so in particular if there is a nowhere zero complex function $f$ such that $\alpha_{1}=f \alpha_{2}$ then the foliations are equivalent.

The simplest examples of symplectic foliations are given by the Lefschetz pencils constructed by Donaldson [9]. A chart $\phi: U \subset M \rightarrow \mathbb{C}^{n}$ will be called adapted at the point $x \in U$ if $\left(\phi_{*}\right)_{x} \omega=\omega_{0}$, where $\omega_{0}$ is the standard symplectic form in $\mathbb{C}^{n}$. A symplectic Lefschetz pencil on a $2 n$-dimensional symplectic manifold $(M, \omega)$ consists of a codimension 4 symplectic submanifold $N \subset M$ and a map $f: M-N \rightarrow \mathbb{C P}^{1}$ such that locally around $N$ there are adapted coordinates $\left(z_{1}, \ldots, z_{n}\right)$ with values in $\mathbb{C}^{n}$ where $f$ is written as $z_{2} / z_{1}$. Also $f$ has finitely many isolated critical points around which there are adapted coordinates where $f=z_{1}^{2}+\cdots+z_{n}^{2}+c$. Finally the fibers of $f$ are symplectic off their singularities. These belong to a special kind of foliations defined as follows:

Definition 1.2 A symplectic foliation $\alpha$ on a $2 n$-dimensional symplectic manifold $(M, \omega)$ is of Kupka type if the singular set $S_{\alpha}$ is a disjoint union of:

$i$. Isolated points where there are adapted charts $\left(z_{1}, \ldots, z_{n}\right)$ such that $\alpha=z_{1} d z_{1}+\cdots+z_{n} d z_{n}$.

ii. Codimension 4 smooth symplectic submanifolds such that each point has an adapted chart $\left(z_{1}, \ldots, z_{n}\right)$ with $\alpha=\eta\left(z_{1}, z_{2}\right)$ for a 1-form $\eta$ of two complex variables with $d \eta(0) \neq 0$ and $\eta^{-1}(0)=\{0\}$.

We want to show a general construction of symplectic foliations

Theorem 1.3 Let $(M, \omega)$ be a symplectic manifold. Then $M$ admits symplectic foliations of Kupka type which are not symplectic Lefschetz pencils. Also $M$ admits symplectic foliations not of Kupka type.

The method of construction is a generalization of the techniques developed in [12]. The structure of the paper is as follows. In section 2 we give the basic results of the theory of holomorphic foliations. Section 3 reviews the asymptotically holomorphic theory introduced in [8] and used in $[2,12]$. 
Next in section 4 we introduce the notion of foliation in this category and check that asymptotically holomorphic foliations with some property of transversality give symplectic foliations. In the following section we move on to prove that it is possible to obtain asymptotically holomorphic foliations by embedding $M$ into the projective space $\mathbb{C P}^{d}$ and intersecting the image with a given holomorphic foliation of $\mathbb{C P}^{d}$. Finally section 6 is devoted to giving some examples of foliations constructed with these techniques.

\section{Codimension one holomorphic foliations}

In this section we discuss briefly the theory of holomorphic foliations on a compact connected complex manifold $M$. A codimension one holomorphic foliation with singularities in $M$ is an equivalence class of holomorphic $\alpha \in$ $H^{0}\left(M, T^{*} M \otimes L\right)$, where $L$ is a holomorphic line bundle and $\alpha \wedge d \alpha=0$.

Given a foliation $\alpha$, we say that $p \in M$ is a regular point if $\alpha(p) \neq 0$. Otherwise, we say that $p$ is singular. The set

$$
S_{\alpha}=\{p \in M \mid \alpha(p)=0\}
$$

is the singular set. If this set has components of codimension 1 , let $D$ be the corresponding divisor. Then there exists a holomorphic section $f$ of $\mathcal{O}(D)$ such that $\alpha / f$ is a foliation whose singularities are of codimension two or more. So we can always suppose that $\operatorname{codim}_{\mathbb{C}} S_{\alpha} \geq 2$.

For a regular point $p \in M$ there exists an open neighborhood $U \subset M$ of $p$ such that $\alpha$ may be written as

$$
\alpha=h d f
$$

in $U$, where $h$ and $f$ are holomorphic functions in $U$. Such $f$ is called first integral and $h$ an integrating factor. The leaves of the foliation in $U$ are the level surfaces of $f$. Globally, the leaves of the foliation $\alpha$ are the leaves of the foliation defined in $M-S_{\alpha}$. If $V$ is a compact hypersurface of $M$ such that $V-V \cap S_{\alpha}$ is a leaf, in general, we have $V \cap S_{\alpha} \neq \emptyset$. In this case, by abuse of language, we will say that $V$ is a compact leaf of the foliation.

\subsection{Kupka singularities}

In this section, we will consider an important class of singularities which have stability properties under deformations.

Definition 2.1 The Kupka singular set of the foliation $\alpha$ consists of the points

$$
K_{\alpha}=\{p \in M \mid \alpha(p)=0, d \alpha(p) \neq 0\}
$$


For every connected component $K \subset K_{\alpha}$, there exists a holomorphic 1-form

$$
\eta=A\left(z_{1}, z_{2}\right) d z_{1}+B\left(z_{1}, z_{2}\right) d z_{2}
$$

called the transversal type at $K$, defined on a neighborhood $V$ of $0 \in \mathbb{C}^{2}$ and vanishing only at 0 , an open cover $\left\{U_{i}\right\}$ of a neighborhood of $K$ in $M$ and a family of submersions $\varphi_{i}: U_{i} \rightarrow \mathbb{C}^{2}$, such that

$$
\varphi_{i}^{-1}(0)=K \cap U_{i}, \quad \text { and }\left.\quad \alpha\right|_{U_{i}}=\varphi_{i}^{*} \eta
$$

A foliation $\alpha$ is of Kupka type if $K_{\alpha}$ is compact and connected.

The main examples of foliations of Kupka type are the following: Let $L_{1}$ and $L_{2}$ be holomorphic line bundles on $M$, such that $L_{1}^{\otimes p}=L_{2}^{\otimes q}$, where $p$ and $q$ are relatively prime, positive integers. Given $f_{1}$ and $f_{2}$ holomorphic sections of the line bundles $L_{1}$ and $L_{2}$ respectively, the holomorphic section

$$
\alpha=p f_{2} d f_{1}-q f_{1} d f_{2} \in H^{0}\left(M, T^{*} M \otimes L_{1} \otimes L_{2}\right),
$$

is a foliation. Moreover the leaves of the foliation represented by $\alpha$, are the fibers of the meromorphic map $\phi=f_{1}^{p} / f_{2}^{q}$. We say that the map $\phi$ is a meromorphic first integral of the foliation represented by $\alpha$.

A branched Lefschetz pencil (a Lefschetz pencil if $p=q=1$ ) is a meromorphic map satisfying the following conditions:

i. The holomorphic line bundles $L_{1}$ and $L_{2}$ are positive.

ii. The hypersurfaces $\left\{f_{1}=0\right\}$ and $\left\{f_{2}=0\right\}$ are smooth, and meet transversely along a codimension two submanifold $K$.

iii. The subvarieties defined by $\lambda f_{1}^{p}-\mu f_{2}^{q}=0$ with $[\lambda: \mu] \in \mathbb{C P}^{1}$, are smooth on $M-K$, except for a finite set of points, where they have just a non-degenerate critical point.

These foliations are of Kupka type with $K_{\alpha}=\left\{f_{1}=f_{2}=0\right\}$.

Theorem 2.2 ([6]) Let $\alpha$ be a foliation of Kupka type in $\mathbb{C P}^{n}, n \geq 3$. $K_{\alpha}$ is a complete intersection if and only if $\alpha=p f_{1} d f_{2}-q f_{2} d f_{1}$.

For foliations on $\mathbb{C P}^{n}, n \geq 6$, it may be shown that any foliation of Kupka type is a branched Lefschetz pencil.

For the unbranched case, we have the following construction involving the fundamental group [4]. Consider a family $\left(E_{t}, \sigma_{t}\right)$ of projectively flat bundles of rank two with section such that $\left(E_{0}, \sigma_{0}\right)=\left(L_{1} \oplus L_{1},\left(f_{1}, f_{2}\right)\right)$ is a Lefschetz pencil. If $L_{1}$ is sufficiently ample, then it is true that $H^{0}\left(E_{t}\right) \neq 0$, so we may consider the foliation $\sigma_{t}^{*} \mathcal{H}_{t}$, where $\mathcal{H}_{t}$ denotes the flat structure on the $\mathbb{C P}^{1}$-bundle $\mathbb{P}\left(E_{t}\right)$.

It is an open question whether any foliation of Kupka type with positive normal bundle and transversal type $z_{2} d z_{1}-z_{1} d z_{2}$ may be described as above. 


\subsection{Logarithmic foliations}

A holomorphic integrating factor of a foliation $\alpha$ is a holomorphic section $\varphi \in H^{0}(M, L)$ such that the meromorphic 1-form $\Omega=\frac{\alpha}{\varphi}$ is closed.

Theorem $2.3([13])$ Let $M$ be a projective manifold with $H^{1}(M ; \mathbb{C})=0$, and let $\varphi=\varphi_{1}^{r_{1}} \cdots \varphi_{k}^{r_{k}}$ be an integrating factor of a foliation $\alpha$. Then

$$
\Omega=\frac{\alpha}{\varphi}=\sum_{i=1}^{k} \lambda_{i} \frac{d \varphi_{i}}{\varphi_{i}}+d\left(\frac{\psi}{\varphi_{1}^{r_{1}-1} \cdots \varphi_{k}^{r_{k}-1}}\right), \quad \text { where } \lambda_{i} \in \mathbb{C}
$$

and $\psi$ is a holomorphic section of the line bundle $\mathcal{O}\left(\sum_{i=1}^{k}\left(r_{i}-1\right)\left\{\varphi_{i}=0\right\}\right)$.

From this equation, we have that the hypersurfaces $D_{i}=\left\{\varphi_{i}=0\right\}$ are compact leaves of the foliation $\alpha$. The residue theorem implies the relation:

$$
\sum_{i=1}^{k} \lambda_{i} \cdot\left[\left\{\varphi_{i}=0\right\}\right]=0 \in H^{2}(M ; \mathbb{C}) .
$$

The integrating factor is reduced if $r_{i}=1$. In this case

$$
\alpha=\varphi_{1} \cdots \varphi_{k}\left(\sum_{i=1}^{k} \lambda_{i} \frac{d \varphi_{i}}{\varphi_{i}}\right)
$$

we say that the foliation is logarithmic. The singular set is the union of $D_{i} \cap D_{j}$ for all possible $1 \leq i<j \leq k$. The Kupka set is

$$
K_{\alpha}=S_{\alpha}-\bigcup_{1 \leq i<j<t \leq k}\left(D_{i} \cap D_{j} \cap D_{t}\right),
$$

and it is therefore not compact for $k \geq 3$.

\section{Asymptotically holomorphic theory}

Let $(M, \omega)$ be a symplectic manifold with $[\omega] / 2 \pi \in H^{2}(M ; \mathbb{R})$ an integer cohomology class. Such a symplectic manifold will be called of integer class. Fix an almost complex structure $J$ compatible with $\omega$ and denote $g(u, v)=$ $\omega(u, J v)$ the associated metric. Let $L \rightarrow M$ be a hermitian line bundle with connection whose curvature is $-i \omega$. The key for a search of symplectic objects is to look for objects which are close to be $J$-holomorphic. The asymptotically holomorphic techniques introduced by Donaldson [8] give a method of construction of such objects by means of increasing the positivity of the curvature of the bundles, which is achieved by twisting with $L^{\otimes k}$ for large $k$. Let us introduce the main notations following $[2,12]$. 
Definition 3.1 A sequence of sections $s_{k}$ of hermitian bundles $E_{k}$ with connections on $M$ is called asymptotically holomorphic if $\left|\nabla^{p} s_{k}\right|=O(1)$ for $p \geq 0$ and $\left|\nabla^{p-1} \bar{\partial} s_{k}\right|=O\left(k^{-1 / 2}\right)$ for $p \geq 1$. The norms are evaluated with respect to the metrics $g_{k}=k g$.

Definition $3.2 A$ section $s_{k}$ of the bundle $E_{k}$ is $\eta$-transverse to 0 if for every $x \in M$ such that $\left|s_{k}(x)\right|<\eta$ then $\nabla s_{k}(x)$ has a right inverse $\theta_{k}$ such that $\left|\theta_{k}\right|<\eta^{-1}$.

This means that at a point $x$ close to the zero set of $s_{k}$ the differential $\nabla s_{k}(x): T_{x} M \rightarrow\left(E_{k}\right)_{x}$ is surjective and that, in the orthogonal to the kernel, this map multiplies the length of the vectors at least by $\eta$. These two conditions guarantee that $Z_{k}=Z\left(s_{k}\right)$ is a submanifold with bounded curvature $R_{Z_{k}}$ (in the metric $g_{k}$ ) uniformly on $k$, and that $T_{x} Z_{k}$ is within distance $O\left(k^{-1 / 2}\right)$ of being a complex subspace of $T_{x} M$. The condition of $s_{k}$ being asymptotically holomorphic implies that $Z_{k}$ is symplectic for large $k$.

Definition 3.3 A sequence of submanifolds $S_{k} \subset M$ is called asymptotically holomorphic if

$$
\angle_{M}\left(T S_{k}, J T S_{k}\right)=O\left(k^{-1 / 2}\right), \quad\left|R_{S_{k}}\right|=O(1) .
$$

The angle $\angle_{M}$ measures the distance, in the grassmannian, between two subspaces [12, definition 3.1]. Thus for $k$ large, any element of a sequence of asymptotically holomorphic submanifolds is symplectic.

Our objective will be to define and construct asymptotically holomorphic foliations. This will be done by embedding our manifold $M$ into a projective space and intersecting it with a holomorphic foliation in it.

Definition 3.4 A sequence of embeddings $\phi_{k}: M \rightarrow \mathbb{C P}^{d}$ is $\gamma$-asymptotically holomorphic for some $\gamma>0$ if it satisfies the following conditions:

i. $d \phi_{k}: T_{x} M \rightarrow T_{\phi_{k}(x)} \mathbb{C P}^{d}$ has a left inverse $\theta_{k}$ of norm less than $\gamma^{-1}$ at every point $x \in M$, i.e., $d \phi_{k}$ multiplies the length of vectors at least by $\gamma$.

ii. $\left|\left(\phi_{k}\right)_{*} J-J_{0}\right|_{g_{k}}=O\left(k^{-1 / 2}\right)$ on the subspace $\left(\phi_{k}\right)_{*} T_{x} M$, where $J_{0}$ is the complex structure of $\mathbb{C P}^{d}$.

iii. $\left|\nabla^{p} \phi_{k}\right|_{g_{k}}=O(1)$ and $\left|\nabla^{p-1} \bar{\partial} \phi_{k}\right|_{g_{k}}=O\left(k^{-1 / 2}\right)$, for all $p \geq 1$.

Theorem 3.5 ([12]) Let $(M, \omega)$ be a closed symplectic $2 n$-dimensional manifold of integer class endowed with a compatible almost complex structure $J$ and let $s_{k}$ be an asymptotically holomorphic sequence of sections of the vector bundles $\mathbb{C}^{d+1} \otimes L^{\otimes k}$ with $d \geq 2 n+1$. Then for any $\alpha>0$ there exists another asymptotically holomorphic sequence $\sigma_{k}$ and $\gamma>0$ such that: 
i. $\left|s_{k}-\sigma_{k}\right|_{C^{2}, g_{k}}<\alpha$.

ii. $\sigma_{k}$ is $\gamma$-projectizable, i.e., $\left|\sigma_{k}\right| \geq \gamma$ on all of $M$, and for all $k$.

iii. $\mathbb{P}\left(\sigma_{k}\right)$ is a $\gamma$-asymptotically holomorphic sequence of embeddings in $\mathbb{C P}^{d}$ for $k$ large enough.

iv. $\phi_{k}^{*}\left[\omega_{F S}\right]=k[\omega]$, where $\omega_{F S}$ is the Fubini-Study symplectic form on $\mathbb{C P}^{d}$.

Moreover, let us have two asymptotically holomorphic sequences $\phi_{k}^{0}$ and $\phi_{k}^{1}$ of embeddings in $\mathbb{C P}^{d}$, with respect to two compatible almost complex structures. Then for $k$ large enough, there exists an isotopy of asymptotically holomorphic embeddings $\phi_{k}^{t}$ connecting $\phi_{k}^{0}$ and $\phi_{k}^{1}$.

The difference with the result stated in [12] is that here we use $C^{2}$-close perturbations, but this makes no real difference. To be able to intersect the embedded manifold with a complex submanifold of $\mathbb{C P}^{d}$ having control of the resulting submanifold, we need a notion of estimated transversality

Definition 3.6 Let $N \subset \mathbb{C P}^{d}$ be a complex smooth submanifold and choose a distribution of complex subspaces $D_{N}(y) \subset T_{y} \mathbb{C P}^{d}$, in a neighborhood of $N$, which extends the tangent distribution to $N$. An embedding $\phi_{k}: M \rightarrow \mathbb{C P}^{d}$ is $\sigma$-transverse to $N$, with $\sigma>0$ small enough, if for all $x \in M$,

$$
d\left(\phi_{k}(x), N\right)<\sigma \Rightarrow L_{m}\left(\left(\phi_{k}\right)_{*}\left(T_{x} M\right), D_{N}\left(\phi_{k}(x)\right)\right)>\sigma .
$$

This angle $L_{m}$ measures the amount of transversality between two intersecting vector subspaces [12, definition 3.3]. The condition above assures that the intersection $\phi_{k}(M) \cap N$ is a sequence of asymptotically holomorphic submanifolds of $M$ (see [12, proposition 3.10]). Moreover, this condition may be achieved.

Theorem $3.7([\mathbf{1 2}])$ Let $\phi_{k}=\mathbb{P}\left(s_{k}\right)$, where $s_{k}$ is a $\gamma$-projectizable asymptotically holomorphic sequence of sections of $\mathbb{C}^{d+1} \otimes L^{\otimes k}, d \geq 2 n+1$, such that $\phi_{k}$ is a $\gamma$-asymptotically holomorphic sequence of embeddings, for some $\gamma>0$. Let $N$ be a complex submanifold in $\mathbb{C P}^{d}$. Then for any $\delta>0$ there exists an asymptotically holomorphic sequence of sections $\sigma_{k}$ of $\mathbb{C}^{d+1} \otimes L^{\otimes k}$ such that

i. $\left|\sigma_{k}-s_{k}\right|_{C^{2}, g_{k}}<\delta$.

ii. $\psi_{k}=\mathbb{P}\left(\sigma_{k}\right)$ is a $\eta$-asymptotically holomorphic embedding in $\mathbb{C P}^{d}$ which is $\varepsilon$-transverse to $N$, for some $\eta>0$ and $\varepsilon>0$, for $k$ large enough.

Moreover, let us have two asymptotically holomorphic sequences $\phi_{k}^{0}$ and $\phi_{k}^{1}$ of embeddings in $\mathbb{C P}^{d}$, with respect to two compatible almost complex structures, which are $\varepsilon$-transverse to $N$. Then for $k$ large enough, there exists an isotopy of asymptotically holomorphic embeddings $\phi_{k}^{t}$ which are $\varepsilon^{\prime}$-transverse to $N$, connecting $\phi_{k}^{0}$ and $\phi_{k}^{1}$. 


\section{Asymptotically holomorphic foliations}

Consider a foliation $\alpha$ of $M$ as an application $\alpha: T M \rightarrow L$. The line bundle $L$ is called the normal bundle of the foliation. Using the almost complex structure on $T M$, we decompose $\alpha$ in complex linear and complex anti-linear parts,

$$
\alpha=\alpha_{1,0}+\alpha_{0,1} \text {. }
$$

When $\alpha_{0,1}(x)=0$ the subspace $\operatorname{ker} \alpha(x) \subset T_{x} M$ is complex. Still when $\left|\alpha_{0,1}(x)\right|<\left|\alpha_{1,0}(x)\right|$ the subspace $\operatorname{ker} \alpha(x)$ is symplectic.

Definition 4.1 A sequence of foliations $\alpha_{k}$ with hermitian normal bundles $E_{k}$ is asymptotically holomorphic if

$$
\begin{gathered}
\left|\nabla^{p} \alpha_{k}\right|=O(1), \quad\left|\nabla^{p}\left(\alpha_{k}\right)_{0,1}\right|=O\left(k^{-1 / 2}\right), \quad p=0,1,2, \\
\left|\nabla^{p-1} \bar{\partial}\left(\alpha_{k}\right)_{1,0}\right|=O\left(k^{-1 / 2}\right), \quad p=1,2 .
\end{gathered}
$$

Also we need a measure of transversality for foliations. This is provided by the following definition. As in the holomorphic case, there is a subset of the singular case, $K_{\alpha_{k}} \subset S_{\alpha_{k}}$ which is easily controlled, and which in some cases reduces to the Kupka set of $\alpha_{k}$.

Definition 4.2 Let $\gamma, \varepsilon>0$. A sequence of foliations $\alpha_{k}$ with hermitian normal bundles $E_{k}$ is $(\gamma, \varepsilon)$-regular if there is a subset $K_{\alpha_{k}}$ of $S_{\alpha_{k}}$ such that

i. $K_{\alpha_{k}}$ is a union of (closed) asymptotically holomorphic submanifolds whose intersections are transverse and asymptotically holomorphic submanifolds.

ii. Let $B_{\gamma}^{k}$ be the tubular neighborhood of radius $\gamma$ of $K_{\alpha_{k}}$ in $g_{k}$-norm. Then $\alpha_{k}$ defines a regular foliation in $B_{\gamma}^{k}-K_{\alpha_{k}}$ such that for any point $x \in B_{\gamma}^{k}-K_{\alpha_{k}}$ it is satisfied that

$$
\angle_{M}\left(\operatorname{ker} \alpha_{k}, J \operatorname{ker} \alpha_{k}\right)=O\left(k^{-1 / 2}\right),
$$

i.e., the leaves in $B_{\gamma}^{k}-K_{\alpha_{k}}$ are asymptotically holomorphic.

iii. $\left(\alpha_{k}\right)_{1,0}$ is $\varepsilon$-transverse to zero as a section of $T^{1,0} M^{*} \otimes E_{k}$ over $M-B_{\gamma}^{k}$.

iv. For any point $x \in M-B_{\gamma}^{k}$, there is a uniform number $r>0$ such that in the ball of $g_{k}$-radius $r$ centered at $x$, the foliation can be written as $\alpha_{k}=h_{k} d f_{k}$, for some trivialization of $E_{k}$, where $h_{k}$ and $f_{k}$ are asymptotically holomorphic and $h_{k}$ is bounded above and below by a uniform constant (independent of $k$ and $x$ ). 
Proposition 4.3 Let $\alpha_{k}$ be a sequence of asymptotically holomorphic foliations with hermitian normal bundles $E_{k}$ which are $(\gamma, \varepsilon)$-regular. Then there exists an arbitrarily small $C^{1}$-perturbation of $\alpha_{k}$ which is a symplectic foliation for $k$ large enough.

Proof. For $k$ large enough, $\alpha_{k}$ defines a symplectic foliation in $B_{\gamma}^{k}$ as a simple corollary of the definition (see [12, section 3.2]). In the complementary $M-B_{\gamma}^{k}$ we study the set of bad points

$$
B\left(\alpha_{k}\right)=\left\{x \in M-B_{\gamma}^{k}||\left(\alpha_{k}\right)_{1,0}(x)|\leq|\left(\alpha_{k}\right)_{0,1}(x) \mid\right\} .
$$

Notice that this is the set of points where the distribution is singular or is not symplectic. We will say that $x \in M-B_{\gamma}^{k}$ is a critical point of $\alpha_{k}$ if $\left(\alpha_{k}\right)_{1,0}(x)=0$. We want to modify the foliation so that $B\left(\alpha_{k}\right)$ only consists of finitely many isolated critical points in which the foliation has a standard model of the form

$$
z_{1} d z_{1}+\ldots+z_{n} d z_{n}
$$

Using lemma 4.4 below we can take $k$ large enough so that the set of bad points is included in a disjoint collection of balls of uniform size centered at the critical points. Then we perturb the foliation in a small neighborhood of the set of critical points, by using proposition 4.5, to obtain a new foliation with $B\left(\alpha_{k}\right)$ equal to the set of critical points and such that in a neighborhood of a critical point it has the form $\alpha_{k}=\sum z_{i} d z_{i}$.

Lemma 4.4 For $k$ large enough the set $B\left(\alpha_{k}\right)$ is contained in a finite set of disjoint balls $B\left(x_{j}, c\right)$ of uniform $g_{k}$-radius $c$ around the critical points $x_{j}$, such that the balls $B\left(x_{j}, 2 c\right)$ are disjoint and contained in $M-B_{\gamma / 2}^{k}$.

Proof. Given a point $x \in B\left(\alpha_{k}\right)$ then the distance of $x$ to the set of critical points is bounded above, for $k$ large enough, by $c$, where $c>0$ is an arbitrarily small uniform constant. Indeed, for $k$ large enough

$$
\left|\left(\alpha_{k}\right)_{1,0}(x)\right| \leq\left|\left(\alpha_{k}\right)_{0,1}(x)\right| \leq C k^{-1 / 2} .
$$

By the $\varepsilon$-transversality,

$$
\nabla\left(\alpha_{k}\right)_{1,0}(x): T M \rightarrow T^{*} M \otimes E_{k}
$$

has an inverse of norm bounded by $\varepsilon^{-1}$. The bounds in the second derivatives of $\alpha_{k}$ allows us to control the radius where the inverse function theorem applies (see [9, lemma 8]). Therefore there must be a zero of $\left(\alpha_{k}\right)_{1,0}$ in a neighborhood of some uniform radius $c$. Again the inverse function theorem implies that the distance between the critical points is bounded independently of $k$. Moreover this $c$ may be made as small as we please just by increasing $k$. 
Proposition 4.5 Let $\alpha_{k}$ be a sequence of asymptotically holomorphic foliations which is $(\gamma, \varepsilon)$-regular. Let $x_{j} \in M-B_{\gamma}^{k}$ be a critical point of $\alpha_{k}$ such that there are asymptotically holomorphic functions $h_{k}$ and $f_{k}$ with $h_{k}$ bounded below and $\alpha_{k}=h_{k} d f_{k}$ on a neighborhood of uniform $g_{k}$-radius $B\left(x_{j}, 2 c\right)$. Then there exists an arbitrarily small $C^{1}$-perturbation of $\alpha_{k}$ supported in $B\left(x_{j}, 2 c\right)$, which is a symplectic foliation in the annulus $B\left(x_{j}, 2 c\right)-$ $B\left(x_{j}, c\right)$ and of the form $z_{1} d z_{1}+\cdots+z_{n} d z_{n}$ in $B\left(x_{j}, c\right)$.

Proof. Given a critical point $x_{j} \in M-B_{\gamma}^{k}$, i.e., $\left(\alpha_{k}\right)_{1,0}\left(x_{j}\right)=0$, write $\alpha_{k}=h_{k} d f_{k}$ as in the statement. Recall that from the proof of lemma 4.4, the constant $c$ can be chosen arbitrarily small, just by increasing the first $k$ satisfying the property. This implies that $\nabla \alpha_{k}(p)$ is "approximately constant" in that ball (by radial parallel transport). We can use an asymptotically holomorphic chart $\Phi_{k}: B_{\mathbb{C}^{n}}(0,1) \rightarrow B_{g_{k}}\left(x_{j}, 2 c\right)$ provided in [2, lemma 3] to trivialize the manifold at a neighborhood of $x_{j}$. Consider $f_{k}, h_{k}$ then as functions of $z_{1}, \ldots, z_{n}$. We may suppose without loss of generality that $f_{k}\left(x_{j}\right)=0$ and that $h_{k}\left(x_{j}\right)=1$.

Since $\left(\alpha_{k}\right)_{1,0}$ is $\varepsilon$-transverse at $x_{j}$ and $\alpha_{k}$ is asymptotically holomorphic, then for $k$ large enough

$$
\partial\left(\alpha_{k}\right)_{1,0}(0): T M \rightarrow T^{*} M \otimes E_{k}
$$

has an inverse of norm bounded by $\left(\varepsilon^{\prime}\right)^{-1}$, i.e., it multiplies the length of vectors by an amount at least $\varepsilon^{\prime}$, for some $\varepsilon^{\prime}$ slightly smaller than $\varepsilon$. Since $\partial\left(\alpha_{k}\right)_{1,0}(0)=\partial \partial f_{k}(0)$ is the complex Hessian of $f_{k}$, we define

$$
H=\frac{1}{2} \sum \frac{\partial^{2} f_{k}}{\partial z_{i} \partial z_{j}}(0) z_{i} z_{j} .
$$

Consider the following foliation in $B\left(x_{j}, 2 c\right)$,

$$
\tilde{\alpha}_{k}=h_{k} d H \text {. }
$$

Here $d H$ is a holomorphic foliation with respect to the standard complex structure $J_{0}$ on the ball. Since this asymptotically holomorphic chart satisfies that the Nihenjius tensor has norm $O\left(k^{-1 / 2}|z|\right)$, we have that $\left(\tilde{\alpha}_{k}\right)_{0,1}=$ $O\left(k^{-1 / 2}|z|\right)$. We have that $f_{k}(0)=H(0)=0, \partial f_{k}(0)=d H(0)=0$ and $\partial \partial f_{k}(0)=\nabla \nabla H(0)$. Since both are asymptotically holomorphic we have that $\left|f_{k}-H\right|=O\left(|z|^{3}+k^{-1 / 2}|z|\right)$. Analogously $\left|\alpha_{k}-\tilde{\alpha}_{k}\right|=O\left(|z|^{2}+k^{-1 / 2}\right)$.

Now let $\beta$ be a bump function such that $\beta(x)=1$ for $x \in B\left(x_{j}, c\right)$, $\beta(x)=0$ for $x \notin B\left(x_{j}, 3 c / 2\right)$ and $|\nabla \beta|=O\left(c^{-1}\right)$. Define the foliation in the whole of $M$,

$$
\hat{\alpha}_{k}=h_{k} d\left(\beta f_{k}+(1-\beta) H\right)=\beta \alpha_{k}+(1-\beta) \tilde{\alpha}_{k}+\nabla \beta\left(f_{k}-H\right) .
$$


We want to prove that outside $x_{j}$,

$$
\left|\left(\hat{\alpha}_{k}\right)_{1,0}\right|>\left|\left(\hat{\alpha}_{k}\right)_{0,1}\right| \text {. }
$$

In $B\left(x_{j}, c\right), \hat{\alpha}_{k}=\tilde{\alpha}_{k}=h_{k} d H=h_{k}\left(\sum a_{i j} z_{i} d z_{j}\right)$. The $\varepsilon$-transversality of $\left(\alpha_{k}\right)_{1,0}$ implies that all the eigenvalues of the symmetric matrix $\left(a_{i j}\right)$ have norm bigger than $\varepsilon^{\prime}$. Therefore $\left|\tilde{\alpha}_{k}\right| \geq \varepsilon^{\prime}|z| / 2$ (for $c$ small). Also $\left|\left(\tilde{\alpha}_{k}\right)_{0,1}\right| \leq$ $C k^{-1 / 2}|z|$, for some constant $C$, so $(4.1)$ holds in $B\left(x_{j}, c\right)$. On $B\left(x_{j}, 3 c / 2\right)-$ $B\left(x_{j}, c\right)$ we have

$$
\left|\hat{\alpha}_{k}-\tilde{\alpha}_{k}\right| \leq\left|\alpha_{k}-\tilde{\alpha}_{k}\right|+|\nabla \beta|\left|f_{k}-H\right|=O\left(c^{2}+k^{-1 / 2}\right) .
$$

In particular, $\left|\left(\hat{\alpha}_{k}\right)_{0,1}\right|=O\left(c^{2}+k^{-1 / 2}\right)$ and $\left|\left(\hat{\alpha}_{k}\right)_{1,0}\right| \geq \varepsilon^{\prime} c / 2-O\left(c^{2}+k^{-1 / 2}\right)$. Taking $c$ small (but uniformly on $k$ ) and then $k$ large enough we have (4.1).

Finally take $\tilde{h}_{k}$ to be equal to $h_{k}$ in $B\left(x_{j}, 2 c\right)-B\left(x_{j}, 3 c / 2\right)$ and equal to 1 in $B\left(x_{j}, c\right)$. Then

$$
\tilde{h}_{k} d\left(\beta f_{k}+(1-\beta) H\right)
$$

satisfies the required properties and in $B\left(x_{j}, c\right)$ it is of the form $\sum a_{i j} z_{i} d z_{j}$. A suitable change of coordinates transforms this into $\sum z_{i} d z_{i}$.

Remark 4.6 Note that the perturbed foliation in the proof above is of the form $\alpha_{k}=h_{k} d f_{k}$ in $B\left(x_{j}, 2 c\right)$, so the integral submanifolds are the level sets $f_{k}=\lambda$. In a small neighborhood of the singularity, the leaves of the foliation are of the form $\sum z_{i}^{2}=\lambda$.

\section{Construction of asymptotically holomorphic foliations}

Once introduced all the asymptotically holomorphic machinery, we are ready to perform our main construction of asymptotically holomorphic foliations, generalizing the ideas contained in [12]. Let $(M, \omega, J)$ be a $2 n$-dimensional symplectic manifold of integer class with a fixed compatible almost complex structure. Let $L \rightarrow M$ be the hermitian line bundle with connection whose curvature is $-i \omega$.

Take any asymptotically holomorphic sequence of sections $s_{k}=\left(s_{k}^{0}, \ldots, s_{k}^{d}\right)$ of $\mathbb{C}^{d+1} \otimes L^{\otimes k}$ such that $\phi_{k}=\mathbb{P}\left(s_{k}\right): M \rightarrow \mathbb{C P}^{d}$ is a sequence of asymptotically holomorphic embeddings with $\phi_{k}^{*} \mathcal{O}(1)=L^{\otimes k}$, whose existence is guaranteed by theorem 3.5.

Now fix a holomorphic foliation $\alpha \in H^{0}\left(\mathbb{C P}^{d}, T^{*} \mathbb{C P}^{d} \otimes \mathcal{O}(N)\right)$ in $\mathbb{C P}^{d}$, such that the singular set $S_{\alpha}$ is a union of smooth complex submanifolds intersecting transversely. There are many examples of such foliations [3]. We want to study the restriction $\alpha_{k}=\phi_{k}^{*} \alpha$ of the foliation $\alpha$ to the sequences of embeddings $\phi_{k}$ and to prove that for suitable choice of embeddings we get asymptotically holomorphic foliations which are $(\gamma, \varepsilon)$-regular. 
Proposition 5.1 Let $\phi_{k}$ be an asymptotically sequence of embeddings of $M$ into $\mathbb{C P}^{d}$ and let $\alpha$ be a foliation as above in the projective space. Then there is a $C^{2}$-close sequence of embeddings $\psi_{k}$ which is $\gamma$-transverse to every submanifold of the singular set $S_{\alpha}$. Then the induced foliation $\alpha_{k}=\psi_{k}^{*} \alpha$ in $M$ is an asymptotically holomorphic foliation and satisfies conditions (i) and (ii) of definition 4.2 with $K_{\alpha_{k}}=\psi_{k}^{-1}\left(\psi_{k}(M) \cap S_{\alpha}\right)$.

Proof. Let $\phi_{k}$ be an asymptotically sequence of embeddings of $M$ into $\mathbb{C P}^{d}$. Write $S_{\alpha}=\cup S_{i}$, where $S_{i} \subset \mathbb{C P}^{d}$ are smooth complex submanifolds of $\mathbb{C P}^{d}$. We may apply theorem 3.7 to perturb $\phi_{k}$ to a $C^{2}$-close sequence of embeddings $\psi_{k}$ which is $\gamma$-transverse to every submanifold $S_{i}$, for some uniform $\gamma>0$. This implies that the submanifold $\psi_{k}(M)$ intersects $S_{i}$ along an asymptotically holomorphic submanifold by [12, proposition 3.10].

Let $\alpha_{k}=\psi_{k}^{*} \alpha$ be the induced foliation in $M$. Then $\alpha_{k}$ is asymptotically holomorphic using the asymptotically holomorphic bounds of $\psi_{k}$ and the holomorphicity of $\alpha$. Note that $\left(\alpha_{k}\right)_{1,0}=\alpha \circ \partial \psi_{k}$ and $\left(\alpha_{k}\right)_{0,1}=\alpha \circ \bar{\partial} \psi_{k}$.

Now $K_{\alpha_{k}}=\psi_{k}^{-1}\left(\psi_{k}(M) \bigcap S_{\alpha}\right)$ is a finite union of asymptotically holomorphic submanifolds and it is included in $S_{\alpha_{k}}$. The $\gamma$-transversality to $S_{i}$ implies that for the points in a neighborhood of radius $\gamma$ of $S_{i}$, the angle between the tangent space of $\psi_{k}(M)$ and the distribution $D_{S_{i}}$ determined by $S_{i}$ is bigger than $\gamma$. Now one may assume that $D_{S_{i}}(x) \subset \operatorname{ker} \alpha(x)$ in a neighborhood of $S_{i}$. We use a linear algebra result [12, proposition 3.5] that says that for $U, V, W$ subspaces of a finite dimensional euclidean vector space with $V \subset W$ it is satisfied that $\angle_{m}(U, V) \leq \angle_{m}(U, W)$. Therefore

$$
\angle_{m}\left(\operatorname{ker} \alpha(x), T_{x} \phi_{k}(M)\right) \geq \angle_{m}\left(D_{S_{i}}(x), T_{x} \psi_{k}(M)\right) \geq \gamma,
$$

for any $x \in \psi^{-1}\left(B_{\gamma}\left(S_{\alpha}\right)\right)$. This implies that the leaves are asymptotically holomorphic in some $B_{c_{o} \gamma}$, for a constant $c_{o}>0$. This gives the sought property (maybe after multiplying $\gamma$ by a suitable uniform constant).

Theorem 5.2 Let $\phi_{k}$ be a sequence of asymptotically holomorphic embeddings of $M$ into $\mathbb{C P}^{d}$. Fix a holomorphic foliation $\alpha \in H^{0}\left(\mathbb{C P}^{d}, T^{*} \mathbb{C P}^{d} \otimes \mathcal{O}(N)\right)$ in $\mathbb{C P}^{d}$ as above. Then there exists an arbitrarily $C^{2}$-close sequence of embeddings $\psi_{k}$ such that $\alpha_{k}=\psi_{k}^{*} \alpha$ is an asymptotically holomorphic sequence of foliations of $M$ with normal bundle $L^{\otimes N k}$, which is $(\gamma, \varepsilon)$-regular for uniform $\gamma, \varepsilon>0$.

Moreover any two such embeddings $\psi_{k}^{i}, i=0,1$, induce isotopic foliations $\alpha_{k}^{i}$, for $k$ large enough.

Proof. Recall that $\phi_{k}=\mathbb{P}\left(s_{k}\right)$ for a $\gamma$-asymptotically holomorphic sequence of sections $s_{k}$ of $L^{\otimes k} \otimes \mathbb{C}^{d+1}$ which is $\gamma$-projectizable. The property of $\phi_{k}$ being $\gamma$-asymptotically holomorphic is open in $C^{1}$-sense, so any small perturbation will still be $\gamma / 2$-asymptotically holomorphic. 
Using proposition 5.1 we may assume that $\phi_{k}$ is already $\gamma$-transverse to (every submanifold in) $S_{\alpha}$ (reducing $\gamma$ if necessary). The property of an asymptotically holomorphic embedding being $\gamma$-transverse to $S_{\alpha}$ is open in $C^{1}$-sense [12, definition 3.11], so any small perturbation will still be $\gamma / 2$ transverse. Denote by $B_{\gamma}^{k}$ the tubular neighborhood of radius $\gamma$ of $K_{\alpha_{k}}=$ $\phi_{k}^{-1}\left(\phi_{k}(M) \bigcap S_{\alpha}\right)$ in $M$. We need to perturb $\phi_{k}$ to a sequence of embeddings such that $\left(\alpha_{k}\right)_{1,0}$ is $\varepsilon$-transverse to zero in $M-B_{\gamma / 2}^{k}$.

We define the following property for sequences of sections $s_{k}$ which are $\gamma / 2$-projectizable and such that $\phi_{k}=\mathbb{P}\left(s_{k}\right)$ is $\gamma / 2$-asymptotically holomorphic and $\gamma / 2$-transverse to $S_{\alpha}: s_{k}$ satisfies the property $\mathcal{P}(\varepsilon, x)$ if $\left(\phi_{k}^{*} \alpha\right)_{1,0}$ is $\varepsilon$-transverse as a section of $T^{*} M \otimes L^{\otimes N k}$ at the point $x$ or else $x \in B_{\gamma / 2}^{k}$. This property is local and open in $C^{2}$-sense (for $\varepsilon$ small).

We want to use the globalization lemma in [2, proposition 3] which states the following: Let $s_{k}$ be asymptotically holomorphic sections of $E_{k}=L^{\otimes k} \otimes$ $\mathbb{C}^{d+1}$. If we can obtain for any point $x \in M$ and any $\delta>0$ an asymptotically holomorphic sequence of sections $\tau_{k, x}$ with Gaussian decay away from $x$ in $C^{r}$-norm and $\left|\tau_{k, x}\right|_{C^{r}, g_{k}}<\delta$ such that $s_{k}+\tau_{k, x}$ satisfies the property $\mathcal{P}(\sigma, y)$ for all $y$ in a ball of uniform radius $B_{g_{k}}(x, c)$, with $\sigma=c^{\prime} \delta\left(\log \left(\delta^{-1}\right)\right)^{-p}$, with $c, c^{\prime}, p$ independent of $k$, then, given any $\delta>0$, there exist, for all large enough $k$, asymptotically holomorphic sections $\sigma_{k}$ of $E_{k}$ such that $\left|s_{k}-\sigma_{k}\right|_{C^{r}, g_{k}}<\delta$ and the sections $\sigma_{k}$ satisfy $\mathcal{P}(\eta, x)$ for all $x \in M$ with $\eta>0$ independent of $k$.

The transversality of $\phi_{k}$ to $S_{\alpha}$ implies that

$$
\phi_{k}\left(M-B_{\gamma / 2}^{k}\right) \subset \mathbb{C P}^{d}-B_{c_{o} \gamma}\left(S_{\alpha}\right)
$$

for some uniform constant $c_{o}>0$. Now fix a finite covering $U_{j}$ of $\mathbb{C P}^{d}-$ $B_{c_{o} \gamma}\left(S_{\alpha}\right)$ such that in each of the sets $U_{j}$ one may write $\alpha=h_{j} d f_{j}$ where $h_{j}$ is a (holomorphic) integrating factor and $f_{j}$ is a first integral.

Let $x \in M-B_{\gamma / 2}^{k}$. We may choose $c$ small enough so that $\phi_{k}\left(B_{g_{k}}(x, c)\right) \subset$ $U_{j}$ for some $j$ (since $\left|\nabla \phi_{k}\right|_{g_{k}} \leq C$ ). Also any small perturbation will still be inside the same open set. Define $f_{k}^{j}=f_{j} \circ \phi_{k}$ and $h_{k}^{j}=h_{j} \circ \phi_{k}$. Both are asymptotically holomorphic in the ball. Moreover $\alpha_{k}=h_{k}^{j} d f_{k}^{j}$. The functions $h_{k}^{j}$ are bounded above and below by fixed constants. Therefore checking transversality for $\left(\alpha_{k}\right)_{1,0}$ is equivalent to checking transversality for $\partial f_{k}^{j}$.

With a transformation of $U(d+1)$ in $\mathbb{C}^{d+1}$ we may suppose that $s_{k}(x)=$ $\left(s_{k}^{0}(x), 0, \ldots, 0\right)$. As $s_{k}$ is $\gamma$-projectizable and asymptotically holomorphic, we suppose that $\left|s_{k}^{0}\right| \geq \gamma / 2$ on $B_{g_{k}}(x, c)$ (maybe reducing $c>0$ ). By $[2$, lemma 2] there are asymptotically holomorphic sections $s_{k, x}^{\text {ref }}$ of $L^{\otimes k}$ with Gaussian decay away from $x$ and with $\left|s_{k, x}^{\text {ref }}\right| \geq c_{1}$ on $B_{g_{k}}(x, c)$, for some uniform $c_{1}>0$. 
We use the standard chart $\Psi_{0}$ in $\mathbb{C P}^{d}$ around $p=\phi_{k}(x)=[1: 0 \cdots: 0]$. With respect to this trivialization the map $\phi_{k}$ is given locally as

$$
\begin{aligned}
\Psi_{0} \circ \phi_{k}: B_{g_{k}}(x, c) & \rightarrow \mathbb{C}^{d} \\
y & \rightarrow\left(\frac{s_{k}^{1}(y)}{s_{k}^{0}(y)}, \ldots, \frac{s_{k}^{d}(y)}{s_{k}^{0}(y)}\right) .
\end{aligned}
$$

Now we can suppose that $\left|\partial f_{j}(0)\right|>c_{2}$, for a universal constant $c_{2}$ since we are well away from $S_{\alpha}$. Also we may suppose that $\partial f_{j}(0)=$ $\left(0,0, \ldots, 0, \frac{\partial f_{j}}{\partial w_{d}}(0)\right)$. Therefore $g_{j}=\frac{\partial f_{j}}{\partial w_{d}}$ is big enough in a small neighborhood.

We trivialize $M$ at a neighborhood of $x$ by using the asymptotically holomorphic charts $\Phi_{k}: B_{\mathbb{C}^{n}}(0,1) \rightarrow B_{g_{k}}(x, c)$ provided in [2, lemma 3]. We denote by $f_{k}^{j}$ and $h_{k}^{j}$ again the corresponding functions defined in a ball of $\mathbb{C}^{n}$, which are asymptotically holomorphic. We define the "approximately orthogonal basis" as in [2]

$$
\mu_{k}^{i}=\partial\left(z_{i} \frac{s_{k, x}^{\mathrm{ref}}}{s_{k}^{0}}\right) .
$$

At $x$ it is an orthogonal basis and all the forms are asymptotically holomorphic. We can use (5.1) to locally trivialize the cotangent bundle. In particular we may write the differential of $g_{j}$ as $\partial g_{j}=\sum g_{j ; i} \mu_{k}^{i}$. Define $t=$ $\left(t_{1}, \ldots, t_{n}\right)$ by the formula

$$
\partial f_{k}^{j}=\sum_{1 \leq l, i \leq n} t_{l}\left(g_{j} \delta_{l i}+g_{j ; i} z_{l} \frac{s_{k, x}^{\mathrm{ref}}}{s_{k}^{0}}\right) \mu_{k}^{i},
$$

where $\left(\delta_{l i}\right)$ is the identity matrix. It is easy to check that $t: B_{\mathbb{C}^{n}}(0,1) \rightarrow \mathbb{C}^{n}$ is asymptotically holomorphic. This is because $\left\{\mu_{k}^{1}, \cdots, \mu_{k}^{n}\right\}$ is close to be an orthogonal matrix, and so all the eigenvalues are bounded below and above by positive uniform constants. Also the matrix in the middle is close to being diagonal in a small ball $B_{g_{k}}(x, c)$, so its eigenvalues are bounded below and above uniformly. Moreover the amount of transversality of $t$ and of $\partial f_{k}^{j}$ are related by non-zero uniform constants. So we need only to get transversality for $t$.

The main local result is Donaldson's theorem 12 in [9] stating that there exists $w=\left(w_{1}, \ldots, w_{n}\right) \in \mathbb{C}^{n}$ with $|w|<\delta$ such that $t-w$ is $\sigma$-transverse to 0 over the ball $B_{\mathbb{C}^{n}}\left(0, \frac{9}{10}\right)$, with $\sigma=\delta\left(\log \left(\delta^{-1}\right)\right)^{-p}$, for a universal $p>0$ and $k$ large enough.

Now define the perturbation

$$
\tau_{k, x}=\left(0, \ldots, 0,-\sum w_{i} z_{i} s_{k, x}^{\mathrm{ref}}\right) .
$$


This is asymptotically holomorphic, with Gaussian decay away from $x$ and norm less than $\delta$. The asymptotically holomorphic sequence $\hat{s}_{k}=s_{k}+\tau_{k, x}$ has corresponding $\hat{t}=t-w+O\left(\delta^{2}\right)$. Let us check this. The embedding associated to $\hat{s}_{k}$ will be denoted by $\hat{\phi}_{k}$ and $\hat{f}_{k}^{j}=f_{j} \circ \hat{\phi}_{k}$. Then

$$
\begin{aligned}
\hat{f}_{k}^{j} & =f_{k}^{j}+d f_{j}\left(\phi_{k}\right)\left(0, \ldots, 0,-\sum w_{i} z_{i} \frac{s_{k, x}^{\mathrm{ref}}}{s_{k}^{0}}\right)+O\left(\delta^{2}\right) \\
& =f_{k}^{j}-\frac{\partial f_{j}}{\partial w_{d}}\left(\sum w_{i} z_{i} \frac{s_{k, x}^{\mathrm{ref}}}{s_{k}^{0}}\right)+O\left(\delta^{2}\right),
\end{aligned}
$$

where the term $O\left(\delta^{2}\right)$ is bounded in $C^{1}$-norm. From this one obtains

$$
\partial \hat{f}_{k}^{j}=\partial f_{k}^{j}-\left(g_{j} \sum w_{i} \mu_{k}^{i}+g_{j ; i} \mu_{k}^{i} \sum w_{l} z_{l} \frac{s_{k, x}^{\mathrm{ref}}}{s_{k}^{0}}\right)+O\left(\delta^{2}\right) .
$$

from where $\hat{t}=t-w+O\left(\delta^{2}\right)$. Now by construction $t-w$ is $\delta\left(\log \left(\delta^{-1}\right)\right)^{-p_{-}}$ transverse to 0 over the ball. It is easy to see that (for $\delta$ small enough) the last term does not affect this property. So the induced $\hat{\alpha}_{k}=\hat{\phi}_{k}^{*} \alpha$ satisfies that $\left(\hat{\alpha}_{k}\right)_{1,0}$ is $C^{\prime} \sigma$-transverse over $B_{g_{k}}(x, c)$, where $C^{\prime}$ is again another uniform constant. This concludes the proof.

For the one-parameter case, let $\psi_{k}^{i}: M \rightarrow \mathbb{C P}^{d}, i=0,1$, be two asymptotically holomorphic sequences of embeddings with respect to two compatible almost complex structures $J_{i}$, which are $(\gamma, \varepsilon)$-regular, for some $\gamma, \varepsilon>0$. Consider a one-parameter family of compatible almost complex structures $J_{t}, t \in[0,1]$, interpolating between $J_{0}$ and $J_{1}$ and let $s_{k}^{t}$ be $J_{t}$-asymptotically holomorphic sections of $L^{\otimes k} \otimes \mathbb{C}^{d+1}$ such that $\psi_{k}^{0}=\mathbb{P}\left(s_{k}^{0}\right)$ and $\psi_{k}^{1}=\mathbb{P}\left(s_{k}^{1}\right)$.

We initially perturb $s_{k}^{t}$ using theorem 3.7 so that all $\psi_{k}^{t}=\mathbb{P}\left(s_{k}^{t}\right)$ are asymptotically holomorphic embeddings which are $\gamma$-transverse to $S_{\alpha}$ (reducing $\gamma>0$ if necessary). Then the argument above works for oneparameter families of sections depending on $t \in[0,1]$ since all the ingredients do (see $[2,9,12]$ ). This means that for a given $\delta>0$, there exists, for large enough $k, J_{t^{-}}$asymptotically holomorphic sections $\sigma_{k}^{t}$ of $L^{\otimes k} \otimes \mathbb{C}^{d+1}$ such that $\left|s_{k}^{t}-\sigma_{k}^{t}\right|<\delta$ and $\mathbb{P}\left(\sigma_{k}^{t}\right)$ are $(\gamma / 2, \eta)$-regular asymptotically holomorphic embeddings in $\mathbb{C P}^{d}$ for some uniform $\eta>0$. Taking $\delta>0$ very small, the linear segment $u s_{k}^{0}+(1-u) \sigma_{k}^{0}, u \in[0,1]$, consists of sections inducing $(\gamma / 2, \varepsilon / 2)$-regular maps. This provides an isotopy $s_{k}^{t}{ }^{t}$ between $s_{k}^{0}$ and $s_{k}^{1}$. The foliations $\alpha_{k}^{t}=\left(\psi_{k}^{\prime t}\right)^{*} \alpha, \psi_{k}^{\prime t}=\mathbb{P}\left(s_{k}^{\prime t}\right), t \in[0,1]$, provide an isotopy between the initial ones, as required.

Remark 5.3 The perturbation of the foliation carried out in section 4 can be done in a one-parameter family $\alpha_{k}^{t}$, as long as we start with a one-parameter family of asymptotically holomorphic functions $f_{k}^{t}$ and $h_{k}^{t}$ to start with. 
Therefore for a family of $(\gamma, \varepsilon)$-regular asymptotically holomorphic foliations $\alpha_{k}^{t}$, there exists a family of symplectic foliations $\hat{\alpha}_{k}^{t}$ interpolating between the perturbations $\hat{\alpha}_{k}^{0}, \hat{\alpha}_{k}^{1}$ of $\alpha_{k}^{0}$, $\alpha_{k}^{1}$ carried out in proposition 4.3. So the construction of the symplectic foliations is unique up to symplectic isotopy, for $k$ large enough.

Remark 5.4 Suppose that $(M, \omega)$ is a symplectic manifold with $[\omega] / 2 \pi$ not an integer cohomology class in $H^{2}(M ; \mathbb{R})$. Choose a compatible almost complex structure $J$. We may take a small perturbation $\omega^{\prime}$ of $\omega$ which is still symplectic and compatible with $J$, such that $\left[\omega^{\prime}\right] / 2 \pi$ is a rational cohomology class. Therefore there is a positive integer $N$ such that $N\left[\omega^{\prime}\right] / 2 \pi$ is an integer cohomology class.

Applying the theorem above for $N \omega^{\prime}$ we get asymptotically holomorphic foliations, and therefore symplectic foliations for $(M, \omega)$, with hermitian normal bundles $L^{\otimes k}$ where $c_{1}(L)=N\left[\omega^{\prime}\right] / 2 \pi$.

\section{Examples}

We can apply all the precedent constructions to any fixed foliation in the projective space. There is a large number of examples $[3,7]$. We are going to compute explicitly some classical cases.

\subsection{Application to Lefschetz pencils}

First, we can recover Donaldson's result [9] on the existence of Lefschetz pencils. We need the following definition.

Definition 6.1 A branched $(p, q)$ Lefschetz pencil, with $p, q>0$ relatively prime, over an oriented closed manifold $M$ consists of the following set of data:

i. A codimension four smooth submanifold $B$.

ii. A map $f: M-B \rightarrow \mathbb{C P}^{1}$ which is a submersion outside a finite set of points $\Delta$.

Also the data fit in the following models:

- Given a point $x \in B$, there exists a neighborhood of $x$ with oriented coordinates $\left(z_{1}, \ldots, z_{n}\right)$ of $M$ where the map $f$ can be written as $f\left(z_{1}, \ldots, z_{n}\right)=z_{2}^{q} / z_{1}^{p}$.

- Given a point $x \in \Delta$, there exists a neighborhood of $x$ with oriented coordinates $\left(z_{1}, \ldots, z_{n}\right)$ of $M$ where the map $f$ can be written as $f\left(z_{1}, \ldots, z_{n}\right)=z_{1}^{2}+\cdots+z_{n}^{2}+c$. 
A branched $(1,1)$ Lefschetz pencil is called a simple Lefschetz pencil (or a Lefschetz pencil, for brevity). The main result in [9] is

Theorem 6.2 Let $(M, \omega)$ be a symplectic manifold of integer class. There exists an integer $k_{0}>0$ such that for any $k>k_{0}, M$ admits a Lefschetz pencil structure $\left(f_{k}, B_{k}, \Delta_{k}\right)$ where all the fibers of the map $f_{k}$ are symplectic and Poincare dual to $k[\omega] / 2 \pi$ and $B_{k}$ is also symplectic.

Donaldson constructs two asymptotically holomorphic sections $s_{k}^{1}, s_{k}^{2} \in$ $L^{\otimes k}$ satisfying certain transversality properties. The map $f_{k}$ is defined by $f_{k}=s_{k}^{2} / s_{k}^{1}$, and for $k$ large, it satisfies the required properties. Moreover, the form $\alpha_{k}=s_{k}^{1} d s_{k}^{2}-s_{k}^{2} d s_{k}^{1}$ is an asymptotically holomorphic symplectic foliation. This follows from the fact that $\alpha_{k}$ is just a rescaling of the differential of $f_{k}$ which is obviously defining a symplectic foliation wherever it is well defined. The rescaling is performed in order to $\alpha_{k}$ be defined all over the manifold. Moreover the foliation is as well symplectic in $B_{k}$, and so it is symplectic all over $M$. We also can prove

Theorem 6.3 Let $(M, \omega)$ be a symplectic manifold of integer class. There exists an integer $k_{0}>0$ such that for any $k>k_{0}, M$ admits a branched $(p, q)$ Lefschetz pencil structure $\left(f_{k}, B_{k}, \Delta_{k}\right)$ where all the fibers of the map $f_{k}$ are symplectic and Poincare dual to $k(p+q)[\omega] / 2 \pi$, and $B_{k}$ is also symplectic.

Proof. Fix sections $s_{1} \in H^{0}\left(\mathbb{C P}^{d}, H^{\otimes q}\right)$ and $s_{2} \in H^{0}\left(\mathbb{C P}^{d}, H^{\otimes p}\right)$, where $H$ is the hyperplane line bundle over $\mathbb{C P}^{d}$. Moreover, suppose that these sections are transverse to zero and $Z\left(s_{1}\right) \cap Z\left(s_{2}\right)$ is a transverse intersection. Therefore

$$
f=\frac{s_{2}^{\otimes q}}{s_{1}^{\otimes p}}
$$

defines a branched $(p, q)$ Lefschetz pencil over $\mathbb{C P}^{d}$. Moreover

$$
\alpha=q s_{1} d s_{2}-p s_{2} d s_{1} \in H^{0}\left(\mathbb{C P}^{d}, T^{*} \mathbb{C P}^{d} \otimes H^{\otimes(p+q)}\right)
$$

is a holomorphic foliation on $\mathbb{C P}^{d}$ satisfying the hypothesis required in section 5 . Then by theorem 5.2, there exists an embedding $\phi_{k}$ of $M$ in $\mathbb{C P}^{d}$ such that $\alpha_{k}=\phi_{k}^{*} \alpha$ is an asymptotically holomorphic and $(\gamma, \varepsilon)$-regular foliation, for uniform $\gamma, \varepsilon>0$. The perturbation performed in proposition 4.3 takes place well away from the singular locus and changes $f_{k}=\phi_{k}^{*} f$ into an integrating function with a suitable form around the critical points. Therefore this perturbation may be done by perturbing either $s_{k}^{1}=\phi_{k}^{*} s_{1}$ or $s_{k}^{2}=\phi_{k}^{*} s_{2}$ (since one of them is non-zero). We obtain a symplectic foliation and the map $f_{k}=\left(s_{k}^{2}\right)^{\otimes q} /\left(s_{k}^{1}\right)^{\otimes p}$ defines a branched $(p, q)$ Lefschetz pencil. 


\subsection{Deformations of Lefschetz pencils with non-trivial holonomy}

We may deform the Lefschetz pencils in the presence of fundamental group as in the algebraic case. We say that a symplectic foliation $\alpha$ on a symplectic manifold $(M, \omega)$ is a deformed Lefschetz pencil if there is a connected smooth codimension four symplectic submanifold $B \subset M$ such that

i. Given a point $x \in B$, there are adapted coordinates $\left(z_{1}, \ldots, z_{n}\right)$ around $x$ where the leaves of the foliation are of the form $z_{2} / z_{1}=\lambda$.

ii. There is a finite set of critical points $x_{j} \in M-B$ such that at any $x_{j}$ there are adapted coordinates $\left(z_{1}, \ldots, z_{n}\right)$ where the leaves of the foliation are of the form $z_{1}^{2}+\cdots+z_{n}^{2}=\lambda$.

Suppose $\alpha$ is a deformed Lefschetz pencil with base locus $B$. The holonomy $H: \pi_{1}(B) \rightarrow \mathrm{PU}(2)$ is defined as follows. Fix $p_{0} \in B$ and consider a small transversal 4-dimensional disk $\Delta$ to $B$. Identify $\mathbb{P}(\Delta)=\mathbb{C P}^{1}$. For any loop $\varsigma$ and any $\lambda \in \mathbb{C P}^{1}$, lift the path $\varsigma$ to a path in a tubular neighborhood of $B$ inside the leaf of $\alpha$ corresponding to the value $\lambda$. The endpoint is defined to be $H(\varsigma)(\lambda) \in \mathbb{C P}^{1}$.

Theorem 6.4 Let $(M, \omega)$ be a symplectic manifold of integer class such that $\operatorname{dim} M=2 n \geq 6$ and $\pi_{1}(M) \neq 1$. Let $\rho: \pi_{1}(M) \rightarrow S U(2)$ be a representation such that $c_{2}\left(E_{\rho}\right)=0$ where $E_{\rho} \rightarrow M$ is the flat $\mathbb{C}^{2}$-bundle corresponding to $\rho$. Let $L \rightarrow M$ be the complex line bundle with $c_{1}(L)=$ $[\omega] / 2 \pi$. Then for $k$ large enough there are deformed Lefschetz pencils $\alpha_{k} \in$ $\mathcal{C}^{\infty}\left(T^{*} M \otimes L^{\otimes k}\right)$ with non-trivial holonomy.

Proof. Under the assumptions of the statement, $E_{\rho}$ is a topologically trivial bundle. So we may understand $E_{\rho}=M \times \mathbb{C}^{2}$ with a flat connection $\nabla_{\rho}=$ $\nabla+\varpi$, where $\varpi \in \Omega^{1}(\mathfrak{s u}(2))$. Now $\left|\nabla_{\rho}-\nabla\right|_{g_{k}}=|\varpi|_{g_{k}}=k^{-1 / 2}|\varpi|_{g}$, i.e., $\nabla_{\rho}$ is an "asymptotically trivial" connection.

Endow $M$ with a compatible almost complex structure and let $L \rightarrow M$ be the hermitian line bundle with connection with curvature $-i \omega$. As in the previous section, there are asymptotically holomorphic sections $s_{k}=\left(s_{k}^{1}, s_{k}^{2}\right)$ of $\mathbb{C}^{2} \otimes L^{\otimes k}$ such that $f_{k}=s_{k}^{2} / s_{k}^{1}$ is a symplectic Lefschetz pencil for $k$ large enough. Let $\alpha_{k}$ be the associated foliation with base locus $B_{k}=Z\left(s_{k}\right)$. Consider the morphism

$$
M-B_{k} \stackrel{\left(1, f_{k}\right)}{\longrightarrow} M \times \mathbb{C P}^{1} \cong \mathbb{P}\left(E_{\rho}\right) .
$$

We pull back the flat distribution of $\mathbb{P}\left(E_{\rho}\right)$ under this map to get a foliation $\alpha_{k}^{\prime}$ in $M$. 
Let $K_{\alpha_{k}}=B_{k}$ be the Kupka set of $\alpha_{k}$ and let $B_{\gamma}^{k}$ be the neighborhood of $g_{k}$-radius $\gamma$ of $K_{\alpha_{k}}$. Then in $M-B_{\gamma}^{k}$ the 1 -form $\left(\alpha_{k}\right)_{1,0}$ is $\varepsilon$-transverse to zero. On the other hand, the horizontal distribution of $M \times \mathbb{C P}^{1}$ is given by $\alpha=d \lambda$, where $\lambda$ is the coordinate in the $\mathbb{C P}^{1}$-direction, and the horizontal distribution for $\mathbb{P}\left(E_{\rho}\right)$ is given by $\alpha^{\prime}=d \lambda+O\left(k^{-1 / 2}\right)$. Since $\alpha_{k}=\left(1, f_{k}\right)^{*} \alpha$ and $\alpha_{k}^{\prime}=\left(1, f_{k}\right)^{*} \alpha^{\prime}$ (up to a factor $\left(s_{k}^{1}\right)^{\otimes 2}$, which is uniformly bounded) we have that $\left|\alpha_{k}-\alpha_{k}^{\prime}\right|_{C^{1}, g_{k}}=O\left(k^{-1 / 2}\right)$. So $\alpha_{k}^{\prime}$ is an asymptotically holomorphic foliation such that $\left(\alpha_{k}^{\prime}\right)_{1,0}$ is $\varepsilon / 2$-transverse to zero in $M-B_{\gamma}^{k}$. After the perturbation in proposition 4.3, it defines a symplectic foliation.

Now we look at $B_{\gamma}^{k}$, where $f_{k}$ has transversal type $z_{1} d z_{2}-z_{2} d z_{1}$. Let us see that this is stable for small perturbations. We construct $E_{\rho}$ in a different way: take the universal covering space $\pi: \tilde{M} \rightarrow M$ and consider $\tilde{M} \times \mathbb{C}^{2}$ with the trivial connection. Identify $(x, f)$ with $(h(x), \rho(h) f)$ for any deck transformation $h$, to get $E_{\rho} \rightarrow M$. Consider the identification of $E_{\rho}$ with the trivial bundle as a (not connection preserving) isomorphism $\psi: \underline{\mathbb{C}}^{2} \rightarrow E_{\rho}$. This lifts to $\tilde{\psi}: \tilde{M} \times \mathbb{C}^{2} \rightarrow \tilde{M} \times \mathbb{C}^{2}$, where $\tilde{\psi}^{*} \nabla=\nabla+\tilde{\varpi}$, $\tilde{\varpi}=\pi^{*} \varpi$. Therefore $\tilde{\varpi}=\tilde{\psi}^{-1} d \tilde{\psi}$ and we may consider $\tilde{\psi}$ as a map $\tilde{M} \rightarrow$ $\mathrm{SU}(2)$ satisfying $\tilde{\psi}(h(x))=\rho(h) \tilde{\psi}(x)$. Look at the map

$$
\tilde{M}-\tilde{B}_{k} \stackrel{\left(1, \tilde{f}_{k}\right)}{\longrightarrow} \tilde{M} \times \mathbb{C P}^{1} \stackrel{\tilde{\psi}}{\cong} \tilde{M} \times \mathbb{C P}^{1},
$$

where $\tilde{f}_{k}=f_{k} \circ \pi$ and $\tilde{B}_{k}=\pi^{-1}\left(B_{k}\right)$. Fix a point $p \in B_{k}$ and $\left(z_{1}, z_{2}, \ldots, z_{n}\right)$ coordinates around $p$ such that $K_{\alpha_{k}}=\left\{z_{1}=z_{2}=0\right\}$ and $f_{k}=z_{2} / z_{1}$. Looking at any point in $\tilde{M}$ over $p$ we see that the leaves of $\alpha_{k}^{\prime}$ are the level sets of $\tilde{\psi} \circ \tilde{f}_{k}$. Denoting

$$
\left(\begin{array}{l}
w_{1} \\
w_{2}
\end{array}\right)=\tilde{\psi}\left(z_{1}, \ldots, z_{n}\right)\left(\begin{array}{c}
z_{1} \\
z_{2}
\end{array}\right)
$$

we have new adapted coordinates $\left(w_{1}, w_{2}, z_{3}, \ldots, z_{n}\right)$ such that the leaves of $\alpha_{k}^{\prime}$ are of the form $w_{2} / w_{1}=\lambda$. Note that the change of coordinates is asymptotically holomorphic, since $|d \tilde{\psi}|_{C^{1}}=O\left(k^{-1 / 2}\right)$.

We have a symplectic foliation $\alpha_{k}^{\prime}$ with $K_{\alpha_{k}}=B_{k}$ a symplectic smooth submanifold of codimension 4 . Let us see that this foliation has non-trivial holonomy (and therefore it is not a Lefschetz pencil). By the Lefschetz theorem in [1], $B_{k}$ is connected and $\pi_{1}\left(B_{k}\right) \rightarrow \pi_{1}(M)$ for $k$ large enough. Therefore $\rho$ defines a non-trivial representation of $\pi_{1}\left(B_{k}\right)$ which determines $\left.\mathbb{P}\left(E_{\rho}\right)\right|_{B_{k}}$. Fix $p \in B_{k}$ and $\Delta$ a small transversal 4-dimensional disk to $B_{k}$ at $p$. Identify $\mathbb{P}(\Delta)=\mathbb{C P}^{1}$. Let $\varsigma \in \pi_{1}\left(B_{k}\right)$ be a loop and $\lambda \in \mathbb{C P}^{1}$. We move $\varsigma$ into a regular leaf starting at the leaf determined by $\lambda$ at $p$. Then looking at the picture in $\tilde{M}$ we see that the end-point is $\rho(\varsigma)(\lambda)$. Note that if 
$\rho\left(\pi_{1}(M)\right) \lambda \subset \mathbb{C P}^{1}$ is infinite then the leaf corresponding to $\lambda$ is not compact. Moreover if $\rho$ is not the identity in $\mathrm{PU}(2)$, then (the closures of) the leaves are not smooth at $B_{k}$ and therefore $\alpha_{k}^{\prime}$ does not define a Lefschetz pencil.

The condition $c_{2}\left(E_{\rho}\right)=0$ is satisfied very often. Since $E_{\rho}$ is a flat bundle, Chern-Weil theory implies that the image of $c_{2}\left(E_{\rho}\right)$ in $H^{4}(M ; \mathbb{R})$ is zero, so $c_{2}\left(E_{\rho}\right)$ is torsion. When $H^{4}(M ; \mathbb{Z})$ is torsion-free, Theorem 6.4 implies that with any representation $\pi_{1}(M) \rightarrow \mathrm{SU}(2)$ whose image is not contained in the center of $\mathrm{SU}(2)$, we can cook a deformed Lefschetz pencil with non-trivial holonomy.

\subsection{Application to asymptotically holomorphic logarithmic folia- tions}

Another useful example of foliations in the projective space are the logarithmic foliations as discussed in subsection 2.2.

Definition 6.5 Let $M$ be a closed manifold, $L_{1}, \ldots, L_{p}$ a family of complex line bundles over $M$ and $\lambda_{1}, \ldots, \lambda_{p} \in \mathbb{C}$ complex numbers such that $\sum \lambda_{i} c_{1}\left(L_{i}\right)=0 \in H^{2}(M ; \mathbb{C})$. Choose sections $f_{1}, \ldots, f_{p}$ of the bundles $L_{1}, \ldots, L_{p}$. Then a logarithmic foliation with normal bundle $L=L_{1} \otimes \cdots \otimes L_{p}$ is given by the twisted 1 -form

$$
\alpha=f_{1} \cdots f_{p} \sum_{i=1}^{p} \lambda_{i} \frac{d f_{i}}{f_{i}} \in \mathcal{C}^{\infty}\left(T_{\mathbb{C}}^{*} M \otimes_{\mathbb{C}} L\right) .
$$

The condition above ensures that in the open subset $\left\{f_{1} \neq 0, \ldots, f_{p} \neq 0\right\}$ we have $\alpha=f_{1} \cdots f_{p} d(\log F)$, where $F=f_{1}^{\lambda_{1}} \cdots f_{p}^{\lambda_{p}}$ is a function. It is easy to see that this gives a well defined $L$-valued 1 -form all over $M$. Now consider the manifold and the sections to be holomorphic. We say that the foliation is generic if:

i. $p \geq 3$ and for any $i=1, \ldots, p$, the line bundle $L_{i}$ is positive.

ii. For any $i=1, \ldots, p$, the hypersurface defined by the equation $\left\{f_{i}=0\right\}$ is irreducible and $\left\{f_{1} \cdots f_{p}=0\right\}$ is a divisor with normal crossings.

In the particular case of the projective space we have that $f_{i} \in H^{0}\left(\mathbb{C P}^{d}, H^{\otimes n_{i}}\right)$ is a homogeneous polynomial of degree $n_{i}$. The condition $\sum \lambda_{i} c_{1}\left(L_{i}\right)=0$ translates into

$$
\sum_{i=1}^{p} n_{i} \lambda_{i}=0
$$

With this kind of foliations at hand we can prove 
Theorem 6.6 Let $(M, \omega)$ be a symplectic manifold of integer class and let $L \rightarrow M$ be a complex line bundle with $c_{1}(L)=[\omega] / 2 \pi$. Given $\left(n_{1}, \ldots, n_{p}\right)$ positive integers and $\left(\lambda_{1}, \ldots, \lambda_{p}\right)$ satisfying the condition (6.1), then for $k$ large enough there exists an asymptotically holomorphic sequence of sections $\left(f_{k}^{1}, \ldots, f_{k}^{p}\right)$ of $L^{\otimes k n_{1}} \oplus \cdots \oplus L^{\otimes k n_{p}}$ such that the associated logarithmic foliation

$$
\alpha_{k}=f_{k}^{1} \cdots f_{k}^{p} \sum_{i=1}^{p} \lambda_{i} \frac{d f_{k}^{i}}{f_{k}^{i}} \in \mathcal{C}^{\infty}\left(T_{\mathbb{C}}^{*} M \otimes_{\mathbb{C}} L^{\otimes k N}\right),
$$

where $N=n_{1}+\cdots+n_{p}$, is a symplectic foliation.

Proof. Choose a generic logarithmic foliation $\alpha$ in the projective space with polynomials $\left(f_{1}, \ldots, f_{p}\right)$ of degrees $\left(n_{1}, \ldots, n_{p}\right)$ and complex numbers $\left(\lambda_{1}, \ldots, \lambda_{p}\right)$ as in the statement. Then the foliation satisfies the conditions of theorem 5.2 , so there is a family of asymptotically holomorphic embeddings in the projective space $\phi_{k}: M \rightarrow \mathbb{C P}^{d}$ such that the pull-back foliations $\phi_{k}^{*} \alpha$ give an asymptotically holomorphic sequence of foliations which are $(\gamma, \varepsilon)$-regular. The perturbation of proposition 4.3 moves the integrating factor $\log \left(\left(f_{k}^{1}\right)^{\lambda_{1}} \cdots\left(f_{k}^{p}\right)^{\lambda_{p}}\right)$, where $f_{k}^{i}=\phi_{k}^{*} f_{i}$. When the critical point is well away from every $D_{k}^{i}=Z\left(f_{k}^{i}\right)$ this perturbation can be absorbed into a perturbation of some $f_{k}^{i}$. To avoid that the critical points get close to $D_{k}^{i}$, just take the embeddings $\phi_{k}$ to be transverse to every $D_{i}=\left\{f_{i}=0\right\} \subset \mathbb{C P}^{d}$ by using theorem 3.7. This produces logarithmic symplectic foliations for $k$ large enough.

Remark that any element of this family of foliations is not equivalent to a Lefschetz pencil. In particular, they are not of Kupka type.

\section{References}

[1] Auroux, D.: Asymptotically holomorphic families of symplectic submanifolds. Geom. Funct. Anal. 7 (1997), 971-995.

[2] Auroux, D.: Symplectic 4-manifolds as branched coverings of $\mathbb{C P}^{2}$. Invent. Math. 139 (2000), 551-602.

[3] Calvo-Andrade, O.: Irreducible components of the spaces of holomorphic foliations. Math. Ann. 299 (1994), 751-767.

[4] Calvo-Andrade, O.: Deformation of the holonomy of a Lefschetz pencil. Qual. Theory Dyn. Syst. 1 (2000), 231-245.

[5] Cerveau, D. and Mattei, J. F.: Formes intégrables holomorphes singulières. Astérisque 97, Société Mathématique de France, Paris, 1982.

[6] Cerveau, D. and Lins Neto, A.: Codimension one foliations in $\mathbb{C P}^{n}$, $n \geq 3$, with Kupka components. Complex Analytic Methods in Dynamical Systems. (Rio de Janeiro, 1992). Astérisque 222 (1994), 93-133. 
[7] Cerveau, D. and Lins Neto, A.: Irreducible components of the space of holomorphic foliations of degree two in $\mathbb{C P}(n), n \geq 3$. Ann. of Math. (2) 143 (1996), 577-612.

[8] Donaldson, S. K.: Symplectic submanifolds and almost-complex geometry. J. Differential Geom. 44 (1996), 666-705.

[9] Donaldson, S. K.: Lefschetz pencils on symplectic manifolds. J. Differential Geom. 53 (1999), 205-236.

[10] Gómez-Mont, X. And Lins Neto, A.: Structural stability of singular holomorphic foliations having a meromorphic first integral. Topology $\mathbf{3 0}$ (1991), 315-334.

[11] McDuff, D. and Salamon, D.: Introduction to symplectic topology. Oxford Mathematical Monographs. Oxford Science Publications. The Clarendon Press, Oxford University Press, New York, 1995.

[12] Muñoz, V., Presas, F. And Sols, I.: Almost holomorphic embeddings in Grassmanians with applications to singular symplectic submanifolds. J. Reine Angew. Math. 547 (2002), 149-189.

[13] Paul, E.: Étude topologique des formes logarithmiques fermées. Invent. Math. 95, 395-420 (1989).

Recibido: 18 de abril de 2002

Omegar Calvo

CIMAT

Ap. Postal 402

Guanajuato 36000, Gto., México

omegar@cimat.mx

Vicente Muñoz

Departamento de Matemáticas

Universidad Autónoma de Madrid

28049 Madrid, Spain

vicente.munoz@uam.es

Francisco Presas

Departamento de Matemáticas

Universidad Autónoma de Madrid

28049 Madrid, Spain

francisco.presas@uam.es

Partially supported by The European Contract Human Potential Programme, Research Training Network HPRN-CT-2000-00101. Third author has conducted his research with a grant from Fundación Pedro Barrié de la Maza. 